\title{
LAS NIIF PARA PYMES-MÓDULO 8: NOTAS A LOS ESTADOS FINANCIEROS
}

\author{
IFRS for SMEs-Module 8: Notes to the Financial Statements. \\ Mario A. Quintero E. \\ Magister, Facultad de Administración de Empresas y Contabilidad, Universidad de Panamá \\ marioquintero@gmail.com
}

\begin{abstract}
Resumen
Las NIIF para las PYMES-Modulo 8: Notas a los estados financieros tema central de este ensayo ha sido de gran utilidad para establecer los parámetros de presentación de los estados financieros y de las notas que deben acompañarlos, para su mayor compresión y mejor uso de la información contable.

También abarcaremos su historia que inicia en los años 50 a 60; la definición de PYMES que no es más que un conjunto de pequeñas y medianas empresas que se miden de acuerdo al volumen de ventas, capital social y el número de personas ocupadas. Sus inicios en Panamá es decir la Resolución №30 - 2010 de 28 de octubre de 2010 donde la junta técnica de contabilidad aprobó la adopción de esta NIIF y definiremos su alcance, estructura y comparación de la NIIF completa con la NIIF para las PYMES.
\end{abstract}

Palabras claves: NIIF, Pymes, estados financieros, medición.

\begin{abstract}
IFRS for SMEs-Module 8: Notes to the financial statements central theme of this essay has been very useful to establish the presentation parameters of the financial statements and the notes that must accompany them, for greater compression and better use of The accounting information.

We will also cover its history that begins in the 50s to 60s; the definition of SMEs that is nothing more than a set of small and medium enterprises that are measured according to the volume of sales, share capital and the number of people employed. Its beginnings in Panama is Resolution No. 30 - 2010 of October 28, 2010 where the technical accounting board approved the adoption of this IFRS and we will define its scope, structure and comparison of the complete IFRS with the IFRS for SMEs.
\end{abstract}

Keywords: IFRS, SMEs, financial statement, measured 


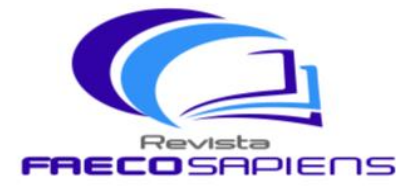

Revista ค月EСロS月คIEחS
ताT Volumen 3 - Número 1

Enero-Junio 2020

Recibido: 23/10/19 Aceptado: 20/11/19 pp. 53-63 Indexada

\section{INTRODUCCIÓN}

Este módulo se centra en la presentación de las notas a los estados financieros de acuerdo con la sección 8 notas a los estados financieros de la NIIF para las PYMES; abordaremos tres opiniones de autores referente a este tema Espejo B., Méndez M. y Zambrano T., Cepeda X. y Auler Michael así como la historia a nivel internacional y local, la definición de PYMES, el alcance, la estructura y comparación de esta NIIF.

Es un tema de gran importancia como bien se explica mediante las opiniones de cada autor presentadas en este ensayo donde se concluye que son parte integral de todos y cada uno de los estados financieros, reflejan información a parte a la que se presenta en los estados financieros mediante relatos detallados para la toma de decisiones de diferente índole.

A las PYMES se las define como un conjunto de pequeñas y medianas empresas que se miden de acuerdo al volumen de ventas, el capital social, el número de personas ocupadas, el valor de la producción o el de los activos. Así también se toma como referencia el criterio económico y el nivel tecnológico. Las PYMES pueden dedicarse a actividades diversas, entre las cuales se pueden mencionar:

- Agricultura, caza, selvicultura y pesca.

- Explotación de minas y canteras.

- Industrias manufactureras.

- Construcción.

- Comercio al por mayor y al por menor, restaurantes y hoteles.

- Transporte, almacenamiento, y comunicaciones.

- Establecimientos financieros, seguros, bienes inmuebles y servicios prestados a las empresas.

- Servicios comunales, sociales y personales. 
Las Normas Internacionales de Información Financiera para Pequeñas y Medianas empresas (NIIF para PYMES) son normas internacionales emitidas por el IASB (International Accounting Standards Board) que se aplican a la elaboración de los estados financieros y otras informaciones financieras pero enfocadas especialmente en Pequeñas y Medianas Empresas.

Las NIIF para PYMES se basan en las NIIF Completas pero con ajustes y modificaciones para reflejar de una manera más adecuada la información financiera y suplir las necesidades de los usuarios de los estados financieros de PYMES.

\section{CONTENIDO}

Las PYMES, comenzaron a adquirir importancia dentro de la economía en los años 50 y 60 con el desarrollo de la revolución industrial con el proceso de tejidos, madera, imprenta, metal, minas y alimentos.

En el 2001 el Consejo de Normas Internacionales de Contabilidad (IASB International Accounting Standard Board) inició un proyecto para desarrollar estándares de contabilidad que sean confiables para las entidades de tamaño pequeño y mediano. Se estableció un grupo de trabajo para ofrecer asesoría sobre los problemas, alternativas y soluciones potenciales.

Desde el inicio del proyecto en el 2001 hasta el 2009, se estuvo en discusiones públicas para desarrollar los estándares de contabilidad internacional para las PYMES.

Y no fue hasta el 9 de julio de 2009 cuando se emitió el IFRS para PYMES. Efectivo cuando sea adoptado por la jurisdicción individual. 


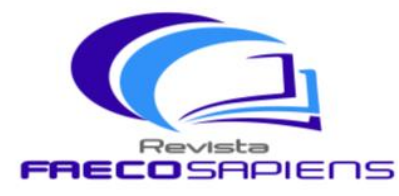

Espejo et al. (2010) señalan que las notas, como presentación de las prácticas contables y revelación de la empresa, son parte integral de todos y cada uno de los estados financieros.

Las notas no son un sustituto del adecuado tratamiento contable en los estados financieros.

Es de gran importancia que la persona que trata de interpretar correctamente un estado financiero, conozca suficientemente sobre asuntos que pueden alterar significativamente los estados financieros como son las políticas de la empresa, los sistemas de inventarios utilizados, los métodos de valuación.

Igualmente es importante que en las notas se consigne lo relacionado con la depreciación de los activos fijos, la valorización que hayan sufrido, los criterios para el manejo de los activos diferidos.

Otro punto importante que debe constar en las notas a los estados financieros, es el tratamiento de ciertos pasivos como es el caso de las provisiones y contingencias, de ingresos para terceros $u$ obligaciones laborales.

Todo estado financiero que se realice, debe contener las notas explicativas respectivas, de tal forma que la persona que los consulte, puede tener la suficiente claridad sobre ellos con el fin que estos puedan cumplir con su verdadero objetivo, cual es el de servir de base para la toma de decisiones, y un estado financiero que no tenga notas aclaratorias y complementarios, no tendrá mayor utilidad.

De acuerdo a, Cepeda (2015) las notas reflejan información a parte a la que se presenta en los Estados Financieros, estas transmiten detalladamente relatos, desagregaciones e información de partidas mostradas en estos estados que no cumplen con las condiciones para ser reconocidas en ellos, de acuerdo con lo 


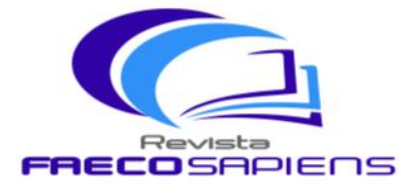

Reviste ค月Eடロら月ค|EПS
ताT Volumen 3 - Número 1

Enero-Junio 2020

Recibido: 23/10/19 Aceptado: 20/11/19 pp. 53-63 Indexada

anterior en casi todas las secciones de las NIIF para PYMES exige información que demuestre que son frecuentemente ilustradas en las respectivas notas.

Con respecto al uso de estimaciones en los estados financieros, una entidad debe revelar la información relacionada con la naturaleza de los supuestos que fueron utilizados, las incertidumbres generadas en la estimación de las partidas en los estados financieros, la susceptibilidad del importe en libros a los métodos y estimaciones implícitas en su cálculo, las soluciones esperadas de las incertidumbres, estas pueden presentar riegos significativos al ocasionar ajustes de importancia en los importes en libros de los activos y pasivos, dentro del siguiente año contable, estas revelaciones mejoran la relevancia, fiabilidad y comprensibilidad de la información presentada en los estados financieros.

En adición, Aular (2018) dice que las notas de los estados financieros y los usuarios de los estados financieros utilizan los mismos para tomar decisiones de diferente índole, como también para evaluar la gestión y situación de la entidad, es por ello que no solo basta contar con las cifras finales presentadas en los estados financieros sino también explicaciones, detalles, juicio profesional, políticas contables, estimaciones y cualquier otra información de utilidad para el lector.

Me he encontrado con muchos gerentes que como lectores les interesa y les parece sumamente útil saber sobre las estimaciones y otros juicios empleados al aplicar las políticas contables, estas revelaciones por ejemplo, les permite a los usuarios de los estados financieros comprender mejor como se aplican las políticas contables, así como hacer comparaciones entre entidades con respecto a los fundamentos sobre los que la gerencia efectúa dichos juicios y estar más involucrados y seguros de la información presentada. 


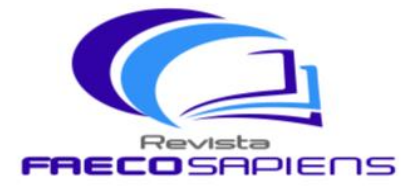

Revista ค月Eடロら月ค|Eחร
ताT Volumen 3 - Número 1

Enero-Junio 2020

Recibido: 23/10/19 Aceptado: 20/11/19 pp. 53-63 Indexada

Sabemos que lo ideal es contar con información cuantitativa y también cualitativa para poder formarnos una opinión y toma de decisiones lo más acertado posible, esto lo entienden muy bien el IASB y le da el rango (digámosle así) a las notas de los estados financieros que merece colocándolo a la par de los estados financieros presentados.

En las notas comúnmente conseguimos antes de la declaratoria de cumplimiento con el estándar la información general de la entidad como lo es su domicilio, sede social, forma legal, país de constitución, descripción de la naturaleza de sus operaciones y principales actividades, la fecha de autorización de los estados financieros para su publicación y quien ha concedido dicha autorización todo esto ayudaran al lector a comprender aún más la entidad.

La información financiera debe ser lo más comprensible posible, y en este hecho las notas son pieza fundamental, las mismas están para facilitar la comunicación efectiva de información en los estados financieros, los gerentes agradecen mucho los detalles y la información adicional relevante que ayude a una mejor comprensión, recordemos que la información presentada es para una amplia gama de usuarios y como tal debemos esforzarnos para presentarla lo más comprensible y útil posible.

\section{CLASIFICACIÓN}

Ley № 33 de 25 de julio de 2000, define el concepto de Micro, Pequeña y Mediana Empresa, sobre la generación del ingreso bruto facturado anualmente:

- Microempresa: Ingresos hasta B/.150,000.

- Pequeña Empresa: De B/.150,001 hasta B/.1,000,000.

- Mediana Empresa: De B/.1,000,001 hasta B/.2,500,000.

Gaceta Oficial Digital, lunes 29 de octubre de 2012. 


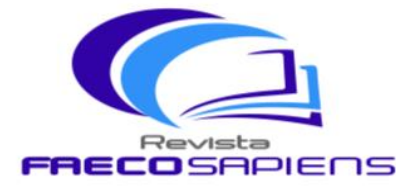

Revista

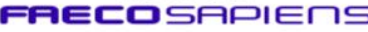

ताT Volumen 3 - Número 1

Enero-Junio 2020

Recibido: 23/10/19 Aceptado: 20/11/19 pp. 53-63 Indexada

Que a través de la Resolución No. 03-2010 de 28 de octubre de 2010 la Junta Técnica de Contabilidad aprobó la adopción de la Norma Internacional de Información Financiera para Pequeñas y Medianas Entidades emitida por el Consejo de Normas Internacionales de Contabilidad como requerimiento en la preparación de estados financieros con propósito general, así como en otra información financiera, para todas aquellas entidades que no cotizan en el mercado de valores o que no tienen obligación pública de rendir cuentas, según está definido en la Norma Internacional de Información Financiera para Pequeñas y Medianas Entidades, a partir del período contable que inicie el 1 de enero de 2011. Queda permitida una adopción anticipada de esta Norma.

\section{CONCLUSIONES}

El alcance de esta norma establece los principios subyacentes a la información a presentar en las notas a los estados financieros y cómo presentarla. Las notas contienen información adicional a la presentada en el estado de situación financiera, estado del resultado integral, estado de resultados (si se presenta), estado de resultados y ganancias acumuladas combinado (si se presenta), estado de cambios en el patrimonio y estado de flujos de efectivo.

La estructura de las notas son las siguientes:

a. Presentarán información sobre las bases para la preparación de los estados financieros, y sobre las políticas contables especificas utilizadas.

b. Revelaran la información requerida por esta NIIF que no se presente en otro lugar de los estados financieros.

c. Proporcionará información adicional que no se presenta en ninguno de los estados financieros, pero que es relevante para la comprensión de cualquiera de ellos. 


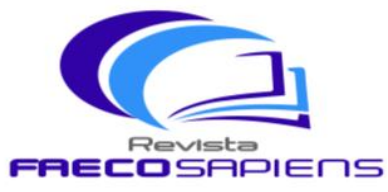

ताT Volumen 3 - Número 1

Enero-Junio 2020

Recibido: 23/10/19 Aceptado: 20/11/19 pp. 53-63

Indexada

Una entidad presentara las notas, en la medida en que sea practicable, de una forma sistemática. Una entidad hará referencia para cada partida de los estados financieros a cualquier información en las notas con la que esté relacionada.

Una entidad presentará normalmente las notas en el siguiente orden:

- Una declaración de que los estados financieros se ha elaborado cumpliendo con la NIIF para las PYMES.

- Un resumen de las políticas contables significativas aplicadas.

- Información de apoyo para las partidas presentadas en los estados financieros en el mismo orden en que se presente cada estado y cada partida.

- Cualquier otra información a revelar.

En comparación con las NIIF completas, no existe diferencia.

Algunas de las informaciones a revelar en las notas:

- Información general.

- Costos financieros.

- Bases de elaboración.

- Inventarios.

- Políticas contables.

- Propiedades, planta y equipo.

- Ingresos de actividades ordinarias.

- Cuentas por pagar.

- Otros ingresos.

- Efectivo y equivalente al efectivo.

- Transacciones entre partes relacionadas.

Algunas de las informaciones a revelar sobre las políticas contables:

- Bases de consolidación.

- Cuentas por pagar.

- Inversiones en asociadas.

- Inventarios.

- Reconocimiento de ingreso de actividades ordinarias.

- Deudores comerciales.

- Costos por préstamos.

- Arrendamientos. 


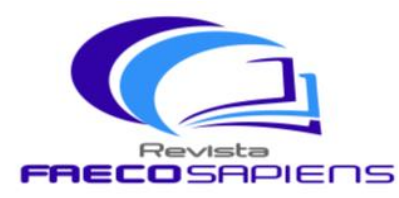

- Propiedades, planta y equipo.

- Activos intangibles.

Algunas de las informaciones a revelar sobre juicios:

- Entidades de cometido específico.

- Baja de consolidación.

- Demanda.

- Clasificación de arredramiento.

Algunas de las informaciones a revelar sobre las fuentes clave de incertidumbre en la estimación:

- Valor razonable de los instrumentos financieros.

\section{CONCLUSIÓN}

Estamos consientes que aun surgirán otras opiniones referentes a la NIIF para PYMES sección 8 Notas a los estados financieros donde se continuara explicando su importancia con respecto a la presentación de los estados financieros resultado que claramente podemos observar con la elaboración de este ensayo; queda entendido la importancia de las notas para la toma de decisiones por lo cual su continuo mejoramiento en la presentación no debe detenerse.

\section{REFERENCIAS BIBLIOGRÁFICAS}

Aular Michael (2018). Las notas a los estados financieros según la NIIF para PYMES. Recuperado de https://www.gestiopolis.com/las-notas-a-los-estados-financierossegun-la-niif-para-pymes/ el 12/8/2019.

Cepeda X. (2015). Preparación de estados financieros y notas a los estados financieros 


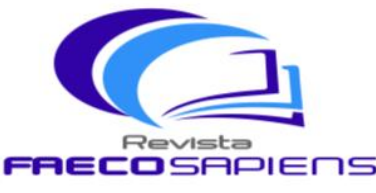

गTV Volumen 3 - Número 1 DRJI

Enero-Junio 2020

Recibido: 23/10/19 Aceptado: 20/11/19 pp. 53-63

Indexada

Recuperado de http://repository.ucc.edu.co/bitstream/ucc/1746/1/Trabajo\%20\%20Preparacion\%20de\%20Estados\%20Financieros\%20y\%20Notas\%20a\%20los\%2 0Estados\%20Financieros\%20-.pdf el 12/8/2019.

Deloitte (2010). EI IFRS para PYMES en su bolsillo. Recuperado de https://www2.deloitte.com/content/dam/Deloitte/pa/Documents/audit/2015/2015-01Pa-Auditoria-NIIFPYMESBolsillo.pdf el 26/8/2019.

Espejo B., Méndez M. y Zambrano T. (2010). NIIF para PYMES: Notas a los estados financieros. Recuperado de http://dspace.ucuenca.edu.ec/handle/123456789/1348?locale=es el 12/8/2019. Fundación IFRS (2009). Módulo 8: Notas a los Estados Financieros. Recuperado de http://crconsultorescolombia.com/wp-content/uploads/2014/10/8 Notas-a-losEstados-Financieros 2013.pdf el 26/8/2019.

Grace Kelly Chi (2012). Publican NIIF para Pymes. Recuperado de https://www.gacetaoficial.gob.pa/pdfTemp/27152 A/39494.pdf el 26/8/2019.

Paz N. (2007). PYME - Panamá. Recuperado de: http://cpc.org.br/pdf/palestrasencontro/Palestra6.pdf el 26/8/2019.

Rodríguez D. (2018). Normas Internacionales de Información Financiera para PYMES. Recuperado de https://contabilidad.com.do/niif-pymes/ el 26/8/2019. 